\title{
Low-Dose Chemoreduction for Infants Diagnosed with Retinoblastoma before 6 Months of Age
}

\author{
Jesse L. Berry ${ }^{a} \quad$ Rima Jubran $^{b} \quad$ Thomas C. Lee $^{a} \quad$ A. Linn Murphree ${ }^{a}$ \\ Diana Lee $^{c}$ Jonathan W. Kim ${ }^{a}$ \\ ${ }^{a}$ The Vision Center, Children's Hospital Los Angeles, USC Eye Institute, and b The Children's Center \\ for Cancer and Blood Diseases, Children's Hospital Los Angeles, University of Southern \\ California Keck School of Medicine, Los Angeles, Calif., and ' ${ }^{\mathrm{C}}$ Georgetown University School \\ of Medicine, Washington, D.C., USA
}

\section{Key Words}

Retinoblastoma · Chemotherapy · Chemoreduction · Treatment outcomes · Infants ·

Neonates $\cdot 6$ months of age

\begin{abstract}
Aim: The purpose of this study was to evaluate the outcomes of infants diagnosed with retinoblastoma before 6 months of age, including the need for chemoreduction (CRD). In this age group, dosage of CRD was reduced due to its potential for toxicity. Methods: This is a retrospective review from 2000 to 2009 that includes 126 eyes of 72 infants (18 unilateral, 54 bilateral). Systemic CRD was administered when local modalities failed or were considered inadequate. Primary outcome measures were the need for CRD and globe salvage. Results: Of the 72 infants diagnosed before 6 months of age, 48 (67\%) ultimately required CRD for globe salvage, 40 (56\%) patients before 6 months of age. Globe salvage was achieved in 62\% (78/126) of eyes overall and in 93\% (68/73) of eyes with Group A-C disease. No patient was hospitalized for CRD-related illness; survival was 100\%. The mean follow-up was 52.9 months (range 1-148 months). Conclusion: Utilizing a combination of focal modalities and reduced-dose CRD, children diagnosed with retinoblastoma before 6 months of age attain globe salvage rates comparable to those of older age groups. Two thirds of the infants ultimately required CRD for globe salvage. Bilateral disease as well as Group D and E classification in at least one eye at presentation increased the chance of requiring CRD $(p<0.0001$ and $p<0.016$, respectively).
\end{abstract}


Berry et al.: Low-Dose Chemoreduction for Infants Diagnosed with Retinoblastoma before 6 Months of Age

\section{Introduction}

The average age at diagnosis for intraocular retinoblastoma is 18 months, 12 months for bilateral patients and 24 months for unilateral patients [1]. However, with improved awareness by pediatricians and parents, many children are being diagnosed with retinoblastoma before 6 months of age [2-5]. These children are more likely to have the hereditary form of retinoblastoma and therefore have a higher likelihood of multiple, bilateral tumors $[2,4]$. For young children diagnosed with retinoblastoma before 6 months of age, there are special treatment-related factors that must be considered. Enucleation can be performed in very young infants but is technically more challenging due to the smaller orbit and the difficulty of placing an adult-sized implant. Radiation was used extensively for globe salvage prior to the 1990s [2, 4], but is now avoided in children younger than 12 months of age due to the significant risk for secondary cancers [6]. Intra-arterial chemotherapy is usually not considered before the child has reached 3 months of age and $6 \mathrm{~kg}$ of weight [7]. Systemic chemoreduction (CRD) has been used successfully worldwide in this age group, but there are concerns regarding its effectiveness in very young infants. This observation may be due to the reduced vascular perfusion and drug delivery in small tumors of young patients [8] as well as the higher risk of side effects, including a possible increased risk for hearing loss [9-11].

At our institution, children diagnosed with retinoblastoma below the age of 6 months are treated with a combination of focal modalities, enucleation, and a modified dose of systemic chemotherapy, depending on the clinical presentation. As outlined by the International Classification System for Retinoblastoma [12], patients with Group A disease in one or both eyes at presentation are managed with focal modalities only. A small minority of eyes with Group B disease with peripheral tumors can be managed with local therapy (i.e. cryotherapy). However, the majority of eyes with Group B disease and all eyes with Group C disease are treated with CRD. The management of Group D disease depends on the laterality: the majority in this age group is treated with CRD; however, enucleation is considered for eyes with Group D disease with poor visual potential, termed Group D2, in unilateral patients [13]. Enucleation is generally recommended for eyes with Group E disease. Chemotherapy for patients below the age of 6 months consists of a 3-drug chemotherapy regimen with carboplatin, etoposide, and vincristine at $50-75 \%$ of the full dose, and vincristine is not given to infants under 2 months of age due to the risk of paralytic ileus. To evaluate the results of young infants with retinoblastoma treated in the CRD era, we retrospectively reviewed outcomes of patients diagnosed with retinoblastoma before 6 months of age treated at Children's Hospital Los Angeles (CHLA).

\section{Materials and Methods}

From January 1, 2000 to December 31, 2009, 310 children were diagnosed with retinoblastoma at CHLA. Within this cohort, 76 infants were diagnosed with retinoblastoma before 6 months of age. At initial diagnosis, there were 20 unilateral patients and 56 bilateral patients (total of 132 eyes). Four patients showed evidence of extraocular disease at diagnosis and were excluded; they were treated according to the Children's Oncology Group protocol for infants with central nervous system disease [14]. Thus, 126 eyes of 72 infants (18 unilateral, 54 bilateral) were included in this retrospective chart review. Primary outcome measures were globe salvage, the need for CRD, and the occurrence of treatment-related complications. The Institutional Review Board at CHLA approved this study.

Treatment

Systemic chemotherapy for patients under 6 months of age consisted of a modified dosing regimen with a $50 \%$ decrease in all agents for the first cycle. At our institution, vincristine is routinely omitted for patients 
Table 1. Chemoreduction protocol at CHLA

\begin{tabular}{|c|c|c|c|c|}
\hline & \multirow[t]{2}{*}{ Standard protocol } & \multicolumn{2}{|c|}{ First cycle: $50 \%$ reduced-dose protocol } & \multirow{2}{*}{$\begin{array}{l}\text { Subsequent cycles: } 50-100 \% \text { dose } \\
\text { protocol }^{\text {a }}\end{array}$} \\
\hline & & for infants $<2$ months & for infants $>2$ months & \\
\hline \multicolumn{5}{|c|}{ Carboplatin } \\
\hline Day 1 & $13 \mathrm{mg} / \mathrm{kg}\left(390 \mathrm{mg} / \mathrm{m}^{2}\right)$ & $6.5 \mathrm{mg} / \mathrm{kg}\left(195 \mathrm{mg} / \mathrm{m}^{2}\right)$ & $6.5 \mathrm{mg} / \mathrm{kg}\left(195 \mathrm{mg} / \mathrm{m}^{2}\right)$ & $6.5-13 \mathrm{mg} / \mathrm{kg}\left(195-390 \mathrm{mg} / \mathrm{m}^{2}\right)$ \\
\hline Day 2 & $13 \mathrm{mg} / \mathrm{kg}\left(390 \mathrm{mg} / \mathrm{m}^{2}\right)$ & $6.5 \mathrm{mg} / \mathrm{kg}\left(195 \mathrm{mg} / \mathrm{m}^{2}\right)$ & $6.5 \mathrm{mg} / \mathrm{kg}\left(195 \mathrm{mg} / \mathrm{m}^{2}\right)$ & $6.5-13 \mathrm{mg} / \mathrm{kg}\left(195-390 \mathrm{mg} / \mathrm{m}^{2}\right)$ \\
\hline \multicolumn{5}{|c|}{ Etoposide } \\
\hline Day 1 & $5 \mathrm{mg} / \mathrm{kg}\left(150 \mathrm{mg} / \mathrm{m}^{2}\right)$ & $2.5 \mathrm{mg} / \mathrm{kg}\left(75 \mathrm{mg} / \mathrm{m}^{2}\right)$ & $2.5 \mathrm{mg} / \mathrm{kg}\left(75 \mathrm{mg} / \mathrm{m}^{2}\right)$ & $2.5-5 \mathrm{mg} / \mathrm{kg}\left(75-150 \mathrm{mg} / \mathrm{m}^{2}\right)$ \\
\hline Day 2 & $5 \mathrm{mg} / \mathrm{kg}\left(150 \mathrm{mg} / \mathrm{m}^{2}\right)$ & $2.5 \mathrm{mg} / \mathrm{kg}\left(75 \mathrm{mg} / \mathrm{m}^{2}\right)$ & $2.5 \mathrm{mg} / \mathrm{kg}\left(75 \mathrm{mg} / \mathrm{m}^{2}\right)$ & $2.5-5 \mathrm{mg} / \mathrm{kg}\left(75-150 \mathrm{mg} / \mathrm{m}^{2}\right)$ \\
\hline \multicolumn{5}{|c|}{ Vincristine } \\
\hline Day 1 & $0.05 \mathrm{mg} / \mathrm{kg}\left(1.5 \mathrm{mg} / \mathrm{m}^{2}\right)$ & not given & $0.025 \mathrm{mg} / \mathrm{kg}\left(0.75 \mathrm{mg} / \mathrm{m}^{2}\right)$ & $0.025-0.05 \mathrm{mg} / \mathrm{kg}\left(0.75-1.5 \mathrm{mg} / \mathrm{m}^{2}\right)^{\mathrm{b}}$ \\
\hline Day 2 & & & & \\
\hline
\end{tabular}

under 2 months of age due to theoretical concerns regarding paralytic ileus. Paralytic ileus is a complication with vincristine therapy [15], although not specifically reported in the pediatric age group. After the first cycle at a 50\% dose of carboplatin, etoposide, and vincristine (or carboplatin and etoposide), patients are monitored for intraocular tumor response and grade III systemic toxicity. If there is adequate tumor response or evidence of systemic toxicity, patients are kept at the $50 \%$ dose for the next cycle. If there is inadequate tumor response and no grade III toxicity then the dose is increased to $75 \%$. Patients can be increased to a $100 \%$ dose of carboplatin, etoposide, and vincristine if all of the following factors are present: (1) older than 3 months of age; (2) no grade III toxicity to the 75\% dose, and (3) inadequate tumor response to the $75 \%$ dose. Standard doses at our institution are as follows: intravenous carboplatin $13 \mathrm{mg} / \mathrm{kg}\left(390 \mathrm{mg} / \mathrm{m}^{2}\right)$ per day for 2 consecutive days, etoposide $5 \mathrm{mg} / \mathrm{kg}\left(150 \mathrm{mg} / \mathrm{m}^{2}\right)$ per day for 2 consecutive days, and vincristine $0.05 \mathrm{mg} / \mathrm{kg}\left(1.5 \mathrm{mg} / \mathrm{m}^{2}\right)$ on the first day only (table 1). Patients with Group B disease are given 3 cycles, patients with Group C disease 4-6 cycles, and those with Group D disease are given 6 cycles (each cycle given 28 days apart).

\section{Chart Review}

At initial evaluation, each patient had a screening MRI of the brain and orbits to evaluate for extraocular disease. Staging examination under anesthesia included intraocular pressure measurements, anterior segment evaluation, dilated fundus examination with scleral depression, B-scan ultrasound, and, for some patients, fluorescein angiography. We classified eyes according to the International Classification System for Retinoblastoma. A chart review was done to obtain the following information: date of birth, date of diagnosis, gender, laterality of retinoblastoma, length of follow-up, systemic chemotherapy agents used with the number of cycles, radiation therapy when utilized, and details of local therapy and visual acuity of the treated eye(s) at the last follow-up visit. Complications of therapy, including National Cancer Institute CTCAE (Common Terminology Criteria for Adverse Events) version 3 (grade III or higher), the occurrence of secondary tumors, metastatic disease, and deaths were also recorded.

\section{Images}

Reviewed images were obtained during examination under anesthesia using a wide-angle contact fundus camera (RetCam; Clarity Medical Systems, Inc., Pleasanton, Calif., USA) and B-scan ultrasound.

\section{Statistical Analysis}

The $\chi^{2}$ test was used for the association between the need for CRD and baseline clinical characteristics (table 2). The primary endpoint in the regression analysis was the need for CRD. In the univariate analysis (table 3), logistic regression was used to assess the significant association of the need for CRD with each listed clinical category. Multivariate analysis using logistic regression analysis was performed to further assess the association with CRD. All results were summarized as odds ratios, 95\% confidence intervals, and $\mathrm{p}$ values. Statistical significance was set at a 2-sided 5\% level. 
Ocular Oncology

and Pathology

Table 2. Demographic and clinical characteristics

\begin{tabular}{|c|c|c|c|}
\hline \multicolumn{4}{|c|}{ Ocul Oncol Pathol 2015;1:103-110 } \\
\hline \multicolumn{2}{|l|}{ DOI: 10.1159/000370215 } & \multicolumn{2}{|c|}{$\begin{array}{l}\text { (C) } 2015 \text { S. Karger AG, Basel } \\
\text { www.karger.com/oop }\end{array}$} \\
\hline \multicolumn{4}{|c|}{$\begin{array}{l}\text { Berry et al.: Low-Dose Chemoreduction for Infants Diagnosed with Retinoblastoma } \\
\text { before } 6 \text { Months of Age }\end{array}$} \\
\hline Characteristics & $\begin{array}{l}\text { No chemotherapy } \\
(\mathrm{n}=24)\end{array}$ & $\begin{array}{l}\text { Chemotherapy } \\
(\mathrm{n}=48)\end{array}$ & $\begin{array}{l}\text { Test of } \\
\text { association } \\
\text { p values }\end{array}$ \\
\hline Gender & & & 0.252 \\
\hline Female & $14(58)$ & $22(46)$ & \\
\hline Male & $10(42)$ & $26(54)$ & \\
\hline Family history & & & 0.580 \\
\hline No & $15(63)$ & $25(52)$ & \\
\hline Yes & $9(38)$ & $23(48)$ & \\
\hline Laterality & & & $<0.0001$ \\
\hline Unilateral & $13(54)$ & $5(10)$ & \\
\hline Bilateral & $11(46)$ & $43(90)$ & \\
\hline $\begin{array}{l}\text { International classi } \\
\text { fication }^{\text {a }}\end{array}$ & & & 0.004 \\
\hline A & $2(8)$ & $3(6)$ & \\
\hline B & $1(4)$ & $10(21)$ & \\
\hline $\mathrm{C}$ & $0(0)$ & $8(17)$ & \\
\hline D & $12(50)$ & $7(14)$ & \\
\hline $\mathrm{E}$ & $9(38)$ & $20(42)$ & \\
\hline Eye classification & & & 0.220 \\
\hline Groups A, B & $3(13)$ & $13(27)$ & \\
\hline Group C or worse & $21(88)$ & $35(73)$ & \\
\hline Eye classification & & & 0.016 \\
\hline Groups A-C & $3(13)$ & $21(44)$ & \\
\hline Group D or worse & $21(88)$ & $27(56)$ & \\
\hline
\end{tabular}

Values are presented as $\mathrm{n}(\%)$. Associations were tested using the $\chi^{2}$ test. ${ }^{\text {a }}$ Patient's most advanced eye.

\section{Results}

From January 1, 2000 to December 31, 2009, 72 infants were diagnosed with intraocular retinoblastoma before the age of 6 months and treated primarily at CHLA. A total of 126 eyes were included in this review. The average age at diagnosis was 2.7 months (range 0-5 months). Table 2 shows the distribution of the demographic and clinical characteristics of our patient population grouped by the need for CRD.

\section{Demographics}

Of the 72 patients, $36(50 \%)$ were female and $36(50 \%)$ were male. A positive family history was noted in $32(44 \%)$ patients. The average age at diagnosis for patients with a positive family history was 2.2 months (range 0-5 months). The 72 patients were classified according to the International Classification System for Retinoblastoma, using the most advanced eye in bilateral cases. Group A disease was diagnosed in 5 (8\%) patients, Group B in 11 (15\%), Group C in 8 (11\%), Group D in 19 (26\%), and Group E in 29 (40\%) patients. At initial diagnosis, 18 (25\%) patients had unilateral disease and 54 (75\%) had bilateral disease. In the unilateral cohort, 4/18 (35\%) patients developed sequential, bilateral disease during follow-up; all 4 patients developed contralateral Group A disease and none required CRD. Of the 4 patients, 3 were diagnosed at 1 month of age and all 4 had a family history of retinoblastoma. 
Ocular Oncology

and Pathology

Table 3. Demographic characteristics associated with the need for systemic CRD

\begin{tabular}{l|l}
\hline Ocul Oncol Pathol 2015;1:103-110 \\
\hline DOI: 10.1159/000370215 & $\begin{array}{l}\text { @ 2015 S. Karger AG, Basel } \\
\text { www.karger.com/oop }\end{array}$ \\
\hline
\end{tabular}

Berry et al.: Low-Dose Chemoreduction for Infants Diagnosed with Retinoblastoma before 6 Months of Age

\begin{tabular}{|c|c|c|c|}
\hline Characteristics & $\begin{array}{l}\text { CRD patients/ } \\
\text { total }\end{array}$ & $\begin{array}{l}\text { Odds ratio } \\
(95 \% \mathrm{CI})\end{array}$ & $\mathrm{p}$ value \\
\hline \multicolumn{4}{|l|}{ Gender } \\
\hline Female & $22 / 36$ & Reference & - \\
\hline Male & $26 / 36$ & $1.77(0.66-4.70)$ & 0.255 \\
\hline \multicolumn{4}{|l|}{ Family history } \\
\hline No & $25 / 40$ & Reference & - \\
\hline Yes & $23 / 32$ & $1.32(0.49-3.56)$ & 0.581 \\
\hline \multicolumn{4}{|l|}{ Laterality } \\
\hline Unilateral & $5 / 18$ & Reference & - \\
\hline Bilateral & $43 / 54$ & $7.60(2.45-23.54)$ & $<0.0001$ \\
\hline \multicolumn{4}{|c|}{ International classification } \\
\hline A & $3 / 5$ & Reference & - \\
\hline B & $10 / 11$ & $6.67(0.44-101.73)$ & 0.172 \\
\hline $\mathrm{C}$ & $8 / 8$ & NA & NA \\
\hline $\mathrm{D}$ & $7 / 19$ & $0.39(0.05-2.92)$ & 0.359 \\
\hline E & $20 / 29$ & $1.78(0.25-12.45)$ & 0.562 \\
\hline \multicolumn{4}{|l|}{ Eye classification } \\
\hline Groups A, B & $13 / 16$ & Reference & - \\
\hline Group C or worse & $35 / 56$ & $0.43(0.11-1.67)$ & 0.223 \\
\hline \multicolumn{4}{|l|}{ Eye classification } \\
\hline Groups A-C & $21 / 24$ & Reference & - \\
\hline Group D or worse & $27 / 48$ & $0.21(0.06-0.80)$ & 0.021 \\
\hline
\end{tabular}


Given the young age of the patients in this series, final Snellen visual acuity could not be determined in all patients; however, $21(30 \%)$ patients had vision better than 20/40 in at least one treated eye at their last recorded follow-up visit.

No patient developed grade III toxicity or higher (CTCAE; absolute neutrophil count $<1,000$ with febrile neutropenia). Granulocyte-stimulating factor support was a component of the chemotherapy regimen and was required for all patients receiving full doses of the 3-drug regimen for CRD. No patients were hospitalized for illness related to CRD. One patient developed severe hearing loss that required hearing aids. Another patient developed nonetoposide-associated AML 12 months after the completion of CRD, and this case has been described previously [17]. All patients were alive and no other secondary malignancies have been identified at a median follow-up of 52.9 months (range 1-148).

\section{Treatment Associations}

Patients are grouped in table 2 by demographic characteristics and whether or not they received CRD. As expected, laterality was significantly associated with requiring CRD. Infants with bilateral disease were 7.60 times more likely to require CRD compared to those with unilateral disease $(\mathrm{p}<0.0001)$. Also, we found a significant association between the International Classification grouping and the need for CRD ( $\chi^{2}$ test, $\left.p=0.004\right)$. For eyes classified as Groups A and B versus those classified as Groups C-E, there is no statistically significant association with the need for CRD $\left(\chi^{2}\right.$ test, $\left.p=0.220\right)$. However, eye classification as Groups A-C versus Groups D and E is significantly associated with the need for CRD ( $\chi^{2}$ test, $p=0.016$ ). The results of the univariate analysis are shown in table 3 . There was no significant association between a positive family history of retinoblastoma and the need for CRD ( $p=0.581$ ). We acknowledge that the need for CRD was made on a clinical basis, and therefore the same results may not apply to other clinical settings.

\section{Discussion}

Treating children diagnosed with retinoblastoma before 6 months of age in the CRD era poses special challenges to the ocular oncology team. Given the potential for an increased risk for complications and systemic toxicity in these young infants, chemotherapy regimens are modified and local therapies are emphasized. As previously reported by Abramson et al. [2], children diagnosed early in life often have advanced disease in one or both eyes. In our series of children diagnosed before the age of 6 months, 42\% (53/126) of eyes were diagnosed with Group D or E disease, similar to a previously published series which included older children [18]. As expected, given the young age at diagnosis, 75\% (54/72) of our patients had bilateral disease. Both of these factors, advanced Group D and E disease and bilateral disease, were associated with the need for systemic CRD ( $p=0.016$ and $p<0.0001$, respectively).

The management of infants diagnosed before the age of 6 months in the CRD era appears to have improved globe salvage and survival rates when compared to previous reports of infants treated predominantly with radiation therapy [2]. With our treatment approach, $33 \%$ of infants diagnosed with retinoblastoma before 6 months of age had no CRD during their treatment course, and $44 \%$ of patients had no CRD during the first 6 months of life. We also demonstrated that a reduced-dose, 2- to 3-drug CRD regimen has acceptable and tolerable systemic drug toxicity even in very young infants. When comparing the rates of systemic toxicity between this reduced-dose approach in young infants versus all children with Group D disease treated with CRD, the rates of grade III toxicity, hospital admissions, hearing loss, and long-term side effects were similar [17]. No patient was admitted for 
chemotherapy-related toxicity during the follow-up period, and only 1 patient developed detectable hearing loss. There were no chemotherapy-related secondary cancers, confirming that even in this age group, avoiding radiation likely avoids secondary malignancies [2, 3]. With our protocol we maintained a 93\% (68/73) salvage rate for eyes with Group A-C disease, a 55\% (6/11) salvage rate for treated eyes with Group D disease, and a 25\% (4/16) salvage rate for eyes with Group E disease. All of these success rates are comparable to standard dose CRD in older age groups $[17,18]$, suggesting that concerns regarding tumor drug resistance with lower dose chemotherapy regimens may be unfounded in this younger group of patients.

The treatment approach for infants diagnosed before the age of 6 months can be particularly challenging in two groups of patients: (1) unilateral patients with Group D disease and (2) asymmetric patients with advanced disease in one eye (Group D) and less advanced disease in the other eye (Groups A, B). For the first group of patients with unilateral Group D disease, we recommend enucleation in most cases, particularly with poor visual potential, to avoid 6 months of systemic chemotherapy in young infants. Sequential, contralateral eye involvement in our group of patients was always Group A disease, which was amenable to treatment with focal modalities without a significant loss of vision. Several authors have previously reported that approximately $20 \%$ of patients before 6 months of age with unilateral disease eventually became bilateral patients $[2,16]$. Abramson et al. [2] recommended that this factor should be considered when making treatment decisions at diagnosis, but we found unconvincing evidence for this treatment concept in our series. Based on our findings, we do not recommend chemotherapy for the fellow eye in this young patient group on a prophylactic or 'protective' basis. For the second group of patients with asymmetric disease, the clinician must make a determination whether the less involved eye can be treated successfully with focal modalities. If not, then the recommended treatment approach is CRD to treat both eyes simultaneously.

\section{Conclusion}

Children diagnosed with retinoblastoma before 6 months of age more often have bilateral disease and are just as likely to have advanced disease as older patients. A treatment approach utilizing local treatment modalities whenever clinically indicated and enucleation for advanced eyes spared many children systemic chemotherapy during the first 6 months of life. When local modalities were inadequate, a reduced-dose CRD protocol with etoposide and carboplatin with or without vincristine was administered with good safety and a tolerable side-effect profile. With this protocol, we report high salvage rates for eyes with Group A-C disease and minimal long-term side effects in retinoblastoma patients diagnosed before 6 months of age.

\section{Acknowledgements}

Statstical consultation was provided by Choo Phei Wee, MA (CHLA). Retinoblastoma International, Inc., the Institute for Families, Inc., Research to Prevent Blindness, and CHLA provided financial and in-kind support.

\section{Disclosure Statement}

No conflicting relationship exists for any author. 
Ocular Oncology

and Pathology
Ocul Oncol Pathol 2015;1:103-110

DOI: $10.1159 / 000370215$

Berry et al.: Low-Dose Chemoreduction for Infants Diagnosed with Retinoblastoma before 6 Months of Age

\section{References}

1 Young JL, Smith MA, Roffers SD, Liff JM, Bunin JR: Retinoblastoma; in: Ries LAG, Smith MA, Gurney JG, Linet M, Tamra T, Young JL, Bunin GR (eds): Cancer Incidence and Survival among Children and Adolescents: United States SEER Program 1975-1995. Bethesda, National Cancer Institute, 1999.

-2 Abramson DH, Notterman RB, Ellsworth RM, Kitchin FD: Retinoblastoma treated in infants in the first six months of life. Arch Ophthalmol 1983;101:1362-1366.

-3 Abramson DH, Du TT, Beaverson KL: (Neonatal) retinoblastoma in the first month of life. Arch Ophthalmol 2002;120:738-742.

4 Abramson DH, Servodidio CA: Retinoblastoma in the first year of life. Ophthalmic Paediatr Genet 1992;13: 191-203.

5 Wong JR, Tucker MA, Kleinerman RA, Devesa SS: Retinoblastoma incidence patterns in the US Surveillance, Epidemiology, and End Results program. JAMA Ophthalmol 2014;132:478-483.

6 Abramson DH, Frank CM: Second nonocular tumors in survivors of bilateral retinoblastoma: a possible age effect on radiation-related risk. Ophthalmology 1998;105:573-579; discussion 579-580.

7 Gobin YP, Dunkel IJ, Marr BP, Francis JH, Brodie SE, Abramson DH: Combined, sequential intravenous and intra-arterial chemotherapy (bridge chemotherapy) for young infants with retinoblastoma. PLoS One 2012; 7:e44322.

-8 Gombos DS, Kelly A, Coen PG, Kingston JE, Hungerford JL: Retinoblastoma treated with primary chemotherapy alone: the significance of tumour size, location, and age. Br J Ophthalmol 2002;86:80-83.

-9 Rizzuti AE, Dunkel IJ, Abramson DH: The adverse events of chemotherapy for retinoblastoma: what are they? Do we know? Arch Ophthalmol 2008;126:862-865.

10 Jehanne M, Lumbroso-Le Rouic L, Savignoni A, et al: Analysis of ototoxicity in young children receiving carboplatin in the context of conservative management of unilateral or bilateral retinoblastoma. Pediatr Blood Cancer 2009;52:637-643.

11 Qaddoumi I, Bass JK, Wu J, et al: Carboplatin-associated ototoxicity in children with retinoblastoma. J Clin Oncol 2012;30:1034-1041.

12 Murphree AL: Intraocular retinoblastoma: the case for a new group classification. Ophthalmol Clin North Am 2005;18:41-53.

13 Berry JL, Jubran R, Wong K, Lee TC, Murphree AL, Kim JW: Factors predictive of long-term visual outcomes of Group D eyes treated with chemoreduction and low-dose IMRT salvage: the Children's Hospital Los Angeles experience. Br J Ophthalmol 2014;98:1061-1065.

14 Armenian SH, Panigrahy A, Murphree AL, Jubran RF: Management of retinoblastoma with proximal optic nerve enhancement on MRI at diagnosis. Pediatr Blood Cancer 2008;51:479-484.

15 Rosenthal S, Kaufman S: Vincristine neurotoxicity. Ann Intern Med 1974;80:733-737.

16 Temming P, Viehmann A, Biewald E, Lohmann DR: Sporadic unilateral retinoblastoma or first sign of bilateral disease? Br J Ophthalmol 2013;97:475-480.

17 Berry JL, Jubran R, Kim JW, et al: Long-term outcomes of Group D eyes in bilateral retinoblastoma patients treated with chemoreduction and low-dose IMRT salvage. Pediatr Blood Cancer 2013;60:688-693.

18 Shields CL, De Potter P, Himelstein BP, Shields JA, Meadows AT, Maris JM: Chemoreduction in the initial management of intraocular retinoblastoma. Arch Ophthalmol 1996;114:1330-1338. 